\title{
Beta-defensin 2 Measurement
}

National Cancer Institute

\section{Source}

National Cancer Institute. Beta-defensin 2 Measurement. NCI Thesaurus. Code C122102.

The determination of the amount of beta-defensin 2 in a biological specimen. 